\title{
A GENERALISATION OF A RESULT OF ABEL WITH AN APPLICATION TO TREE ENUMERATIONS
}

\author{
by M. M. ROBERTSON
}

(Received 12th March 1964)

\section{Introduction}

We prove the following theorem, which was established by Abel (1) for the case $u=1$. then

Theorem. If $u, k$ are positive integers and $x, \alpha_{1}, \ldots, \alpha_{u}, \beta$ are real numbers,

$\left(x+\sum_{j=1}^{u} \alpha_{j}\right)^{k}=\sum_{l=0}^{k} \sum_{s_{1}, \ldots, s_{u}}^{(l)} \frac{k !}{(k-l) ! s_{1} ! \ldots s_{u} !}(x+l \beta)^{k-l} \prod_{i=1}^{u} \alpha_{i}\left(\alpha_{i}-s_{i} \beta\right)^{s_{i}-1}, \ldots$

where the sum $\sum_{s_{1}, \ldots, s_{u}}^{(l)}$ is taken over all distinct ordered solutions $\left(s_{1}, \ldots, s_{u}\right)$ in non-negative integers of the equation $\sum_{i=1}^{\mu} s_{i}=l$.

It is clear that, when $\beta=0$, equation (1) reduces to the multinomial expansion. The theorem is applied in Section 3 to obtain a proof by induction of the well-known result of Cayley that the number of rooted trees with $n$ distinct nodes is $n^{n-1}$.

\section{Proof of the Theorem}

The theorem is proved by induction on $k+u$. It is trivial to verify that (1) holds for $k=1$ and all $u$. In (1) Abel showed that (1) is true for $u=1$ and all $k$. We assume that (1) holds for $k=m-1, u=v$, for $k=m-1, u=v-1$ and for $k=m, u=v-1$, and we prove that then (1) holds for $k=m, u=v$.

By the hypothesis then, we have

$$
\begin{aligned}
\left(x+\sum_{j=1}^{v} \alpha_{j}\right)^{m-1}=\sum_{l=0}^{m-1} \sum_{s_{1}, \ldots, s_{v}}^{(l)} \frac{(m-1) !}{(m-l-1) ! s_{1} ! \ldots s_{v} !}(x+l \beta)^{m-1-1} \\
\times \prod_{i=1}^{0} \alpha_{i}\left(\alpha_{i}-s_{i} \beta\right)^{s_{i}-1} .
\end{aligned}
$$

Integrating with respect to $x$, we obtain

$$
\begin{aligned}
\left(x+\sum_{j=1}^{v} \alpha_{j}\right)^{m}=\sum_{l=0}^{m-1} \sum_{s_{1}, \ldots, s_{v}}^{(l)} \frac{m !}{(m-l) ! s_{1} ! \ldots s_{v} !} & (x+l \beta)^{m-l} \\
& \times \prod_{i=1}^{v} \alpha_{i}\left(\alpha_{i}-s_{i} \beta\right)^{s_{i}-1}+C,
\end{aligned}
$$


where $C$ is independent of $x$. Multiplying (2) by $m \beta$, adding to (3) and then substituting $x=-m \beta$, we obtain

$$
\begin{aligned}
C= & m \beta\left(\sum_{j=1}^{v} \alpha_{j}-m \beta\right)^{m-1}+\left(\sum_{j=1}^{0} \alpha_{j}-m \beta\right)^{m} \\
= & m \beta \sum_{l=0}^{m-1} \sum_{s_{1}, \ldots, s_{v-1}}^{(l)} \frac{(m-1) !}{(m-l-1) ! s_{1} ! \ldots s_{v-1} !}\left\{\alpha_{v}-(m-l) \beta\right\}^{m-l-1} \\
& +\sum_{l=0}^{m} \sum_{s_{1}, \ldots, s_{v-1}}^{(l)} \frac{m !}{(m-l) ! s_{1} ! \ldots s_{v-1} !} \prod_{i=1}^{v-1} \alpha_{i}\left(\alpha_{i}-s_{i} \beta\right)^{s_{i}-1} \\
= & \sum_{l=0}^{m} \sum_{\left.s_{1}, \ldots, s_{v-1}-(m-l) \beta\right\}^{m-l} \prod_{i=1}^{v-1} \alpha_{i}\left(\alpha_{i}-s_{i} \beta\right)^{s_{t}-1}}^{(m-l) ! s_{1} ! \ldots s_{v-1} !} \alpha_{v}\left\{\alpha_{v}-(m-l) \beta\right\}^{m-1-1} \prod_{i=1}^{v-1} \alpha_{i}\left(\alpha_{i}-s_{i} \beta\right)^{s_{i}-1} \\
= & \sum_{s_{1}, \ldots, s_{v}}^{(m)} \frac{m !}{s_{1} ! \ldots . s_{v} !} \prod_{i=1}^{v} \alpha_{i}\left(\alpha_{i}-s_{i} \beta\right)^{s_{i}-1} .
\end{aligned}
$$

This relation in conjunction with (3) completes the proof by induction.

\section{Enumeration of Rooted Trees}

We now prove by induction that the number of rooted trees with $n$ distinct nodes is $n^{n-1}$. As there is only one rooted tree with one node, the formula holds for $n=1$. We assume that the number of rooted trees with $i$ nodes is $i^{i-1}$ for all $i \leqq n$. Now, rooted trees with $n+1$ nodes are formed by first choosing any one of the $n+1$ nodes as root and joining it in any one of $\left(\begin{array}{l}n \\ r\end{array}\right)$ ways to $r$ of the other nodes. The remaining $n-r$ nodes are divided into $r$ ordered sets (some of which may be empty), each of which forms a tree with one of the previous $r$ nodes as root. Therefore, the number of rooted trees with $n+1$ nodes is equal to

$$
(n+1) \sum_{r=1}^{n}\left(\begin{array}{l}
n \\
r
\end{array}\right) \sum_{s_{1}, \ldots, s_{r}}^{(n-r)} \frac{(n-r) !}{s_{1} ! \ldots s_{r} !}\left(s_{1}+1\right)^{s_{1}-1} \ldots\left(s_{r}+1\right)^{s_{r}-1}
$$

and this is equal to $(n+1)^{n}$ whenever, for $1 \leqq r \leqq n$,

$$
\left(\begin{array}{l}
n \\
r
\end{array}\right) \sum_{s_{1}, \ldots, s_{r}}^{(n-r)} \frac{(n-r) !}{s_{1} ! \ldots s_{r} !}\left(s_{1}+1\right)^{s_{1}-1} \ldots\left(s_{r}+1\right)^{s_{r}-1}=\left(\begin{array}{c}
n-1 \\
r-1
\end{array}\right) n^{n-r},
$$

i.e. whenever

Now,

$$
\sum_{s_{1}, \ldots, s_{r}}^{(n-r)} \frac{(n-r) !}{s_{1} ! \ldots s_{r} !}\left(s_{1}+1\right)^{s_{1}-1} \ldots\left(s_{r}+1\right)^{s_{r}-1}=r n^{n-r-1} .
$$

$$
\frac{(n-r) !}{s_{1} ! \ldots s_{r} !}=\frac{(n-r-1) !}{s_{1} ! \ldots s_{r} !} \sum_{l=1}^{\infty} s_{l}=\sum_{l=1}^{r} \frac{(n-r-1) !}{s_{1} ! \ldots s_{l-1} !\left(s_{l}-1\right) ! s_{l+1} ! \ldots s_{r} !},
$$




\section{A GENERALISATION OF A RESULT OF ABEL}

where every $s_{1}>0$. When some $s_{1}$ are zero the corresponding terms are omitted in the final summation of (5). Therefore the left side of (4) is equal to

$$
\begin{gathered}
\sum_{l=1}^{r} \sum_{s_{1}, \ldots, s_{r}}^{(n-r-1)} \frac{(n-r-1) !}{s_{1} ! \ldots s_{r} !}\left(s_{1}+1\right)^{s_{1}-1} \\
\quad \ldots\left(s_{l-1}+1\right)^{s_{l}-1-1}\left(s_{l}+2\right)^{s_{1}}\left(s_{l+1}+1\right)^{s_{l}+1-1} \ldots\left(s_{r}+1\right)^{s_{r}-1} \\
=r \sum_{s_{1}, \ldots, s_{r}}^{(n-r-1)} \frac{(n-r-1) !}{s_{1} ! \ldots s_{r} !}\left(s_{1}+1\right)^{s_{1}-1} \ldots\left(s_{r-1}+1\right)^{s_{r}-1-1}\left(s_{r}+2\right)^{s_{r}}
\end{gathered}
$$

and so, from (4), the formula is verified if

$$
\sum_{s_{1}, \ldots, s_{r}}^{(n-r-1)} \frac{(n-r-1) !}{s_{1} ! \ldots s_{r} !}\left(s_{1}+1\right)^{s_{1}-1} \ldots\left(s_{r-1}+1\right)^{s_{r-1}-1}\left(s_{r}+2\right)^{s_{r}}=n^{n-r-1} .
$$

This relation follows from (1) by putting $u=r-1, k=n-r-1, x=n-r+1$, $\alpha_{1}=\ldots=\alpha_{n}=1, \beta=-1$, and so the proof is complete.

\section{REFERENCE}

(1) N. H. ABEL, Beweis eines Ausdruckes, von welchem die Binomial-Formel ein einzelner Fall ist, Journal für die Reine und Angewandte Mathematik, 1 (1826), 159-160

Department of Mathematics

UNIVERSITY OF CALIFORNIA

Los ANGELES, U.S.A. 